\title{
Early Detection of Oral Pre-Cancerous Lesions: Recent Advances
}

\author{
Anita Meena ${ }^{1}$ and Sujata K. Satoskar ${ }^{2}$ \\ ${ }^{1}$ Department of Conservative Dentistry and Endodontics, Government Dental College \& Hospital, Jaipur Rajasthan, India \\ ${ }^{2}$ Assistant Professor Department of Oral Medicine, Diagnosis, and Radiology Government Dental College \& Hospital Bambolim-Goa, \\ India 403202
}

\section{A BSTRACT}

The high mortality rate in cancer such as oral squamous cell carcinoma is commonly attributed to the negligence in detection of the disease at an early treatable stage. A number of promising recent technologies have been proposed to improve the effectiveness of early oral cancer detection. The goal of a cancer-screening is to detect tumors at a stage early enough so that treatment is likely to be successful. Screening tools are needed that exhibit the combined features of high sensitivity and specificity. Moreover, the screening tool must be sufficiently noninvasive and inexpensive to allow widespread applicability. This paper will attempt to overview the recent advances in the dentistry with special emphasis on detection of oral cancer in early stages and also aim to familiarize, the various diagnostic tools among undergraduates' and postgraduates' from different medical fields.

Keyword: Precancerous lesion, Nanotechnology, Heterozygosity, Saliva

\section{INTRODUCTION}

Oral cancer is the sixth most common cancer in the world. It is a major oncological problem in the regions of the world, where tobacco habits in the form of chewing and/or smoking with or without alcohol intake are common. It typically occurs in the elderly men during the fifth through eighth decade of life and is rarely seen in young people. It constitutes $17 \%$ of all cancers in males and $10.5 \%$ of all cancers in females making it, the commonest cancer in males and the third commonest cancer among females. ${ }^{1}$

Significant development of biotechnology and improvement in our basic understanding of the cancer initiation and progression now enable us to identify tumor signatures, such as oncogenes and tumorsuppressor gene alterations, in bodily fluids that drain

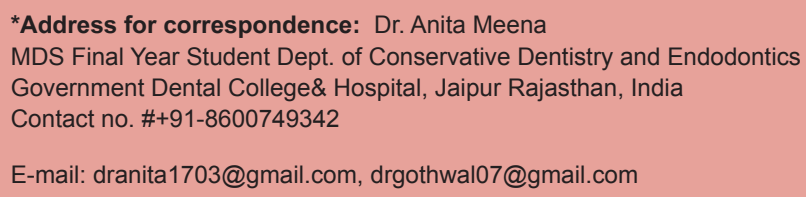

DOI: 10.5530/ijmedph.2.3.1 from the organs affected by the tumor. A number of promising recent technologies have been proposed to improve the effectiveness of early oral cancer detection. These include the use of saliva as an oral cancer screening platform; improved methods of exfoliative cytology, molecular analyses and gene alterations study; use of fluorescent light and other imaging modalities. In this paper, the authors have tried to highlight such techniques and methods for the detection of oral cancer in an early stage, therefore improving the lifestyle of the patients.

\section{RECENT ADVANCES IN DETECTION OF ORAL CANCER}

Conventional methods of detection of oral cancers include conventional biopsy, vital tissue staining, and cytological techniques. Toluidine blue (TB) staining is one of the most widely used, simple and inexpensive diagnostic procedure, which uses a blue dye to highlight abnormal areas of mucosa. ${ }^{2}$ Recent advances for earlier detection of oral cancer and precancerous conditions in the oral cavity widely include the use of following techniques: 
ViziLite-ViziLite ${ }^{\circledR}$ system (Zila Pharmaceuticals, Phoenix, AZ) became the first system approved by the FDA to improve the visualisation of early cancer lesions in head and neck examinations. ${ }^{3}$ In this, the patient performs a one-minute mouthrinse with the diluted acetic acid solution to remove the glycoprotein barrier and then the mucosa dried. The intensity of ambient light is then dimmed and a diffuse bluish-white chemiluminescent light is applied. Normal cells absorb the light and have a bluish colour, whereas the light is reflected by abnormal cells with a higher nucleus : cytoplasm ratio and by epithelium with excessive keratinisation, hyperpara-keratinisation and/or significant inflammatory infiltrate, which appear acetowhite with brighter, more marked and more distinguishable borders. ${ }^{4,5}$

\section{Velscope-VELscope ${ }^{\circledR}$ system (Visually Enhanced} Lesion Scope; LED Dental Inc., White Rock, B.C.) is a simple manual device developed by LED Medical Diagnostics in association with scientists of the British Columbia Cancer Agency (BCCA). It detects the loss of fluorescence in visible and non-visible high-risk oral lesions by applying direct fluorescence. The loss of fluorescence reflects a complex mixture of alterations to the intrinsic tissue distribution of fluorophores. ${ }^{5} \mathrm{It}$ consists of a source of light that emits a wave length of 400 to $460 \mathrm{~nm}$ and a manual unit for direct visualisation. Under this light, normal oral mucosa emits a green auto-fluorescence, whereas abnormal areas absorb the fluorescent light and appear dark. Hence, early biochemical changes are detected before their more evident appearance, permitting the early detection of pathological lesions.

Other improved methods of exfoliative cytology such oral brush biopsy that assures an adequate biopsy sample, which is scanned by Oral CDX computer system specifically designed to detect oral epithelial cancerous \& precancerous lesions. ${ }^{6}$

\section{GENE ALTERATIONS AND MOLECULAR ANALYSES:}

Approximately two-thirds of all head and neck cancer cells contain a deleted region located in chromosome 9p21-22, which appears in dysplastic and carcinoma in situ (CIS) lesions, thereby suggesting that a gene in this region is knocked out early in oral carcinogenesis. Therefore, analyses of molecular biology thus help in early detection of oral cancers. ${ }^{7}$ Use of auxiliary methods such as DNA image cytometry, AgNOR analysis (AgNORs represent silver-stainable nucleolar organizer regions), and multimodal cell analysis has been shown to significantly increase diagnostic accuracy of oral cytology. These methods are only applied on those samples that reveal doubtful or suspicious (dysplastic) cells, on neither cytologically normal nor frankly malignant ones.

Molecular techniques also includes, quantification of nuclear DNA content by flow cytometric analysis, assessing for presence of tumor markers which may be genomic, proliferative \& differential markers. Microsatellite markers are used for studying clonal changes in premalignant lesions \& report loss of chromosomal regions that contain putative tumor suppressor genes. This is termed as loss of heterozygosity $(\mathrm{LOH})$. Frequent occurrence of $\mathrm{LOH}$ is seen at $9 \mathrm{p} \& 3 \mathrm{p}$ regions, which is indicative of progression of dysplasia. Loss of heterozygosity at 4q, 8p, 11q, 13q \& 17p indicates significantly shorter progression time to squamous cell carcinoma. The advantage of microsatellite markers is that they can be assessed on exfoliated cells of oral mucous membrane. $^{8}$

Another recent advance in early detection of oral cancer is Diaelectrophoresis (DEP) technique. DEP is non-invasive method to determine electrophysiological parameters such as conductivity and permittivity of cellular cytoplasm and membrane. Broche L M, Lewis M P, and Porter S (2007) concluded that DEP can be utilised to characterise oral squamous cell carcinoma-derived cells (H357), and human keratinocyte cells and revealed that there are significant differences in these parameters between malignant and normal epithelial cell lines. Results from above mentioned study suggest that DEP has potential for the early detection of cancerous from non-cancerous cells in a clinical setting.?

The molecular markers for oral squamous cell carcinoma show variance in xenobiotic metabolizing enzymes as glutathione $\mathrm{S}$ transferase, glutathione peroxidase (GPX), cytochrome p450, $\mathrm{N}$ acetyl transferase and alcohol dehydrogenase which are involved in detoxification of carcinogens. Mutation in Ras gene, overexpression of Cyclin- D1, Ki-67, activated extracellular signal regulated kinase are overexpressed in cancerous regions while loss of p16 \& p27 are true oncogenic events \& predispose the patient to develop oral squamous cell carcinoma (OSCC). Metalloproteinases \& tissue inhibitors of metalloproteinases (MMP \& TIMP), plasminogen activator inhibitor-1 allele, and gelsolin expression promote cancer initiation \& development in the initial stages. Diagnosis is facilitated by altered expression of Bcl-2, Ki-67, E-cadherin, and $\mathrm{LOH}$ at $9 \mathrm{p} 21 \& 17 \mathrm{p} 13$ result in increased susceptibility to develop OSCC..$^{10}$ 
SALIVARY DIAGNOSTIC TOOLS: Saliva has been found to contain 309 proteins, normal salivary transcriptome contains 3000 mRNA, out of which 180 mRNAs are common in all healthy subjects, they constitute the normal salivary transcriptome core (NSTC). Four genes from this NSTC, which code for IL-8, ornithine decarboxylase, Spermidine acetyltransferase, \& IL-1 $\beta$ are higher in saliva of patients suffering from oral squamous cell carcinoma. ${ }^{11}$ Results from the study conducted by Brailo V, Boras V. V, and Arambasin A.C (2006) showed that significant increased levels of IL- 6 \& TNF- $\alpha$ were found in saliva of patients with precancerous lesions particularly oral leukoplakia. ${ }^{12}$

Microelectromechanical and Nanoelectromechanical systems (MEMS \& NEMS) have been made that exhibit good sensitivity and specificity for analyte detection up to the single molecular level. The envisioned product is called Oral Fluid Nanosensor Test (OFNASET); it is a handheld automated device which enables simultaneous \& rapid detection of multiple salivary proteins and nucleic acids. ${ }^{13}$ The Oral Fluid Nano Sensor Test (OFNASET) technology platform combines cuttingedge technologies, such as self-assembled monolayers (SAM), bionanotechnology, cyclic enzymatic amplification, and microfluidics, with several well-established techniques including microinjection molding, hybridization-based detection, and molecular purification. The intended use of the OFNASET is for the point of care multiplex detection of salivary biomarkers for oral cancer.

Gau V and Wong D (2007) demonstrated that the combination of two salivary proteomic biomarkers (Thioredoxin and IL-8) and four salivary mRNA biomarkers (SAT, ODZ, IL-8, and IL-1b) can detect oral cancer with high specificity and sensitivity. Their preliminary studies had showed compelling results. Therefore, in future this in-office technique will enable detection of oral cancerous lesions, by using a drop of saliva. ${ }^{13,14}$

Nanotechnology is a group of Emerging technologies in which the structure of matter is controlled at the nanometer scale, the scale of small numbers of atoms, to produce novel materials and devices that have useful and unique properties. The various nanoproducts undergoing clinical trials include Lipid based nanoparticles, Quantum dots, Nanoshells, Gold Nanoparticles, Paramagnetic Nanoparticles and Carbon Nanotubes. Gold nanoparticles conjugated to antibody to epidermal growth factor receptor expressed by neoplastic cells binds preferentially to cancerous cells hence making detection easier. ${ }^{14}$ Another non-invasive technique of detection of oral dysplasia is optical coherence tomography (OCT). OCT permits highresolution imaging of tissue surfaces and subsurfaces, with the potential capability for detection and mapping of epithelial pathologies. OCT is very useful tool for the early detection and diagnosis of oral lesions as well as regular monitoring of suspect lesions in the oral cavity and rapid, low-cost screening of high-risk populations. ${ }^{15}$

\section{CONCLUSION}

Early referral and collaboration with dental professionals and owing to these various advances, we will be able to provide quick and efficient care to our patients, thus improving their quality of life. Early diagnosis and treatment results in the prevention of certain health hazards due to radiations and also reduce the possibility of further complications.

ACKNOWLEDGEMENT: The authors are grateful to Dr. Amit Gothwal, Post Graduate Student in Oral Medicine, Diagnosis \& Radiology Department, Government Dental College \& Hospital, Goa (India) for his contribution in providing related articles and his constant support.

\section{REFERENCES}

1. Iype EM, Pandey M, Mathew A, Thomas G, Sebastian P, Nair MK. Oral cancer among patients under the age of 35 years. J Postgrad Med 2001;47:171.

2. Sankaranarayan R, Mohideen MN, Nair MK, Padmanabhan TK,. Aetiology of oral cancer in patients < 30 years of age. Br J Cancer 1989; 59: 439-40.

3. Ram S, Siar $\mathrm{CH}$. Chemiluminescence as a diagnostic aid in the detection of oral cancer and potentially malignant epithelial lesions. Int J Oral Maxillofac Surg. 2005;34:521-7.

4. Farah CS, McCullough MJ. A pilot case control study on the efficacy of acetic acid wash and chemiluminescent illumination (ViziLite) in the visualisation of oral mucosal white lesions. Oral Oncol. 2007;43:820-4.

5. Epstein JB, Silverman S Jr, Epstein JD, Lonky SA, Bride MA. Analysis of oral lesion biopsies identified and evaluated by visual examination, chemiluminescence and toluidine blue. Oral Oncol. 2008;44:538-44.

6. Dinkar A, Satoskar S. Diagnostic aids in early oral cancer detection-a review. JIAOMR, 2006; 18(2), 82-9.

7. Wong DTW, Todd R, Tsuji T and Donoff RB. Molecular Biology of Human Oral Cancer., Crit Rev Oral Biol Med; 1996:7(4):319-28.

8. Wong DT,. Salivary diagnostics powered by nanotechnologies, proteomics \& genomics. J Am Dent Assoc. 2006;137(3):313-21.

9. Broche LM, Lewis MP, Porter S. Early detection of oral cancer using Diaelectrophoresis. Oral Oncology 2007, Vol 43, 199-203.

10. Scully C, Bagán JV. Recent advances in oral oncology; Oral Oncology 2007;43:107-15. 
11. Lee JM, Garon E and Wong DT. Salivary Diagnostics; Orthod Craniofac Res. 2009;12(3): 206-11.

12. Brailo V, Boras V. V, Arambasin A.C,. The significance of salivary interleukin 6 and tumor necrosis factor alpha in patients with oral leukoplakia. Oral Oncology; 2006;42:370-3.

13. Gau V, Wong D. Oral fluid nanosensor test (OFNASET) with advanced electrochemical-based molecular analysis platform. Ann NY Acad Sci 2007;1098:401-10.
14. James Chen Yong Kah, Kiang Wei Kho, CarolineGuat Leng Lee, ColinJames Richard, Sheppard, ZeXiang Shen, Khee Chee Soo, and MaliniCarolene Olivo. Early diagnosis of oral cancer based on the surface plasmon resonance of gold nanoparticles. Int J Nanomedicine. 2007; 2(4): 785-98.

15. Petra Wilder-Smith, Kenneth Lee, Shuguang Guo, Jun Zhang, Kathryn Osann, Zhongping Chen, and Diana Messadi. In Vivo Diagnosis of Oral Dysplasia and Malignancy Using Optical Coherence Tomography: Preliminary Studies in 50 Patients Lasers Surg Med. 2009; 41(5): 353-7. 